\title{
Searching for the Donor Stars of ULX Pulsars
}

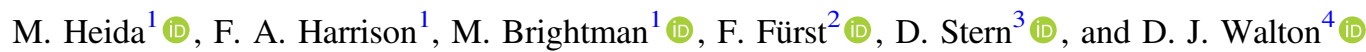 \\ ${ }^{1}$ Cahill Center for Astronomy and Astrophysics, California Institute of Technology, 1200 E. California Blvd, Pasadena, CA 91125, USA; mheida@ caltech.edu \\ ${ }^{2}$ European Space Astronomy Centre (ESAC), Science Operations Department, E-28692 Villanueva de la Cañada, Madrid, Spain \\ 3 Jet Propulsion Laboratory, California Institute of Technology, 4800 Oak Grove Dr, Mail Stop 169-221, Pasadena, CA 91109, USA \\ ${ }^{4}$ Institute of Astronomy, Madingley Rd, Cambridge CB3 0HA, UK \\ Received 2018 October 2; revised 2018 December 18; accepted 2018 December 19; published 2019 February 4
}

\begin{abstract}
We report on our search for the optical counterparts of two ultraluminous X-ray pulsars with known orbital periods, M82 X-2 and NGC 5907 X-1, in new and archival Hubble Space Telescope observations, in an effort to characterize the donor stars in these systems. We detect five near-infrared sources consistent with the position of M82 X-2 that are too bright to be single stars. We also detect seven sources in the WFC3/UVIS F336W image whose photometry matches that of $10-15 M_{\odot}$ stars turning off the main sequence. Such stars have densities consistent with the properties of the donor star of M82 X-2 as inferred from X-ray timing analysis, although it is also possible that the donor is a lower-mass star below our detection limit or that there is a significant contribution from the accretion disk to the optical emission. We detect three candidate counterparts to NGC 5907 X-1 in the near-infrared. All of these are too bright to be the donor star of the ultraluminous X-ray source (ULX), which, based on its orbital period, is a red giant. The high background at the location of NGC 5907 X-1 precludes us from detecting this expected donor star. The recently discovered NGC 5907 ULX-2 also falls within the field of view of the near-infrared imaging; we detect four sources in the error circle, with photometry that matches asymptotic giant branch stars. The star suggested to be the counterpart of NGC 5907 ULX-2 by Pintore et al. falls outside our $2 \sigma$ error circle.
\end{abstract}

Key words: infrared: stars - stars: neutron - X-rays: individual (M82 X-2, NGC 5907 X-1, NGC 5907 ULX-2)

\section{Introduction}

Ultraluminous X-ray sources (ULXs) are off-nuclear pointlike sources with $L_{\mathrm{X}} \geqslant 10^{39} \mathrm{erg} \mathrm{s}^{-1}$, exceeding the Eddington limit for a $10 M_{\odot}$ black hole (e.g., Fabbiano 1989). Although early papers on ULXs suggested intermediate-mass black holes $\left(10^{2} \lesssim M_{\mathrm{BH}} \lesssim 10^{5} M_{\odot}\right)$ as the accretors in these systems (e.g., Colbert \& Miller 2005), a growing body of evidence-mainly from broadband X-ray spectroscopy-favors "stellar-mass" objects exceeding the Eddington limit for the majority of sources (Gladstone et al. 2009; Sutton et al. 2013b; Walton et al. 2018). A combination of super-Eddington accretion and geometric collimation would then explain the large luminosities. This interpretation was proven to be correct for several sources by the discovery of X-ray pulsations from M82 X-2 (Bachetti et al. 2014), and more recently from three other ULXs (NGC 5907 X-1, NGC 7793 P13, and SN2010da, also designated NGC 300 ULX1; Fürst et al. 2016; Israel et al. 2017a, 2017b; Binder et al. 2018; Carpano et al. 2018), showing that in these objects the accretor is in fact a neutron star. X-ray luminosities exceeding $10^{39} \mathrm{erg} \mathrm{s}^{-1}$ have also been observed in outbursts from Be X-ray binaries with neutron star accretors (e.g., SMC X-3 and Swift J0243.6+6124, Tsygankov et al. 2017; Jaisawal et al. 2018; Wilson-Hodge et al. 2018). A fifth neutron star ULX (M51 ULX8), without X-ray pulses but identified through a cyclotron resonance feature, was reported by Brightman et al. (2018). For a recent review on ULXs we refer the reader to Kaaret et al. (2017).

Accreting magnetized neutron stars can, in principle, reach these super-Eddington luminosities through a number of mechanisms. For example, magnetic fields $\gtrsim 10^{12} \mathrm{G}$ will collimate the accretion flow, allowing material to accrete onto the polar regions while radiation escapes from the sides of the column. Strong surface magnetic fields $\left(\gtrsim 10^{13.5} \mathrm{G}\right)$ reduce the scattering cross section for electrons, reducing the radiation pressure and increasing the effective Eddington luminosity (Basko \& Sunyaev 1976; Herold 1979; Mushtukov et al. 2015). However, configurations with low magnetic fields have also been proposed (e.g., Kluzniak \& Lasota 2015). All accretion theories trying to explain the seemingly super-Eddington luminosities of ULXs - with either neutron star or stellar-mass black hole accretors-include a highly super-Eddington mass transfer rate from the donor star, which is difficult to reach through wind accretion. The favored scenario is therefore mass transfer through Roche-lobe overflow from a massive donor star.

Very few mass donors of ULXs have been identified. The large distances to ULXs in combination with their often bright accretion discs make their donor stars hard to detect, and even more difficult to confirm spectroscopically. The only systems where spectroscopic signatures of donor stars are detected are M101 ULX1, with a Wolf-Rayet donor star (Liu et al. 2013); three ULXs (NGC 253 J004722.4-252051, NGC 925 J022721 +333500 . and NGC $4136 \mathrm{~J} 120922+295559)$ with M-type supergiant donors (Heida et al. 2015, 2016); and the ULX pulsar (ULXP) NGC 7793 P13, which has a blue supergiant (B9Ia) donor (Motch et al. 2011, 2014). The recently discovered ULXP SN2010da in NGC 300 likely has a supergiant (sg) B[e] or yellow sg donor star (Lau et al. 2016; Villar et al. 2016). These are all massive stars, confirming the idea that many ULXs are an extreme class of high-mass X-ray binaries and possible progenitors of gravitational-wave sources (Esposito et al. 2015; Inoue et al. 2016; Marchant et al. 2017). However, there is a significant observational bias in favor of massive companions because they are much brighter than lowmass stars. Wiktorowicz et al. (2017) predicted that the 
Table 1

List of HST Observations

\begin{tabular}{|c|c|c|c|c|c|}
\hline Target & Prop ID & Obs Date & Inst. & Filter & Exp. Time (s) \\
\hline \multirow[t]{3}{*}{ NGC 5907} & 15074 & 2017 Dec 13 & WFC3/IR & F160W & 5612 \\
\hline & 6092 & $1996 \mathrm{Mar} 31$ & WFPC2 & F450W & 780 \\
\hline & 6092 & 1996 Mar 31 & WFPC2 & F814W & 480 \\
\hline \multirow{3}{*}{ M82 } & 11360 & 2010 Jan 1 & WFC3/UVIS & F336W & 1620 \\
\hline & 11360 & 2010 Jan 1 & WFC3/IR & F110W & 1195 \\
\hline & 11360 & 2010 Jan 1 & WFC3/IR & F160W & 2395 \\
\hline
\end{tabular}

majority of neutron star ULXs have low-mass $\left(\leqslant 1.5 M_{\odot}\right)$, red giant donors.

For ULXs with a black hole accretor, phase-resolved spectroscopic observations of the donor star are the only direct way to obtain limits on the mass of the compact object. In ULXPs we can turn this around: because neutron stars have been observed to have a very narrow range of masses (1-2 $M_{\odot}$, see Lattimer 2012, for a review), the mass of the compact object is known, and-unless the system is viewed at a very low inclination-we can obtain the orbital parameters of the system by observing the modulation of the pulse period due to the Doppler effect. This gives us the mass function and therefore limits on the mass and average density of the donor star. In combination with direct observations of the donor it is then possible to investigate if these systems are indeed fed through Roche-lobe overflow, as was recently shown for NGC 7793 P13 (Fürst et al. 2018). It is also a potential way to identify ULXs with low-mass donor stars.

In this paper we analyze new and archival Hubble Space Telescope (HST) observations of M82 X-2 and NGC 5907 $\mathrm{X}-1$. We combine the photometry with the orbital parameters of these systems derived from X-ray timing analyses by Bachetti et al. (2014) and Israel et al. (2017a) to put constraints on their respective donor stars. All magnitudes in this paper are Vega magnitudes.

\section{Data Reduction and Analysis}

\subsection{HST Observations}

We obtained a deep HST WFC3/IR F160W image of NGC 5907 (proposal ID 15074) and downloaded archival WFPC2 images of NGC 5907 (Kissler-Patig et al. 1999) and WFC3 images of M82 (e.g., Lim et al. 2013; see Table 1). There are additional deep WFPC2/F606W images of NGC 5907, but NGC $5907 \mathrm{X}-1$ is just outside the field of view. The WFPC2/F450W and F814W observations of NGC 5907 have previously been analyzed by Sutton et al. (2012), who initially reported a candidate counterpart to NGC $5907 \mathrm{X}-1$ that is only detected in the F450W image $\left(m_{\mathrm{F} 450 \mathrm{~W}}=21.5 \pm 0.4\right)$. However, upon closer inspection, this source appeared to be spurious: it is only present in two of the three exposures with the F450W filter and likely due to cosmic rays that unfortunately hit at the same location in these two exposures (Sutton et al. 2013a). The observations of M82 were used by Voss et al. (2011) and Wang et al. (2015) to search for counterparts to M82 X-1, but not M82 X-2. Gladstone et al. (2013) did search for counterparts to M82 X-2 in older HST data (they call the source NGC 3034 ULX4) but they did not detect any candidate counterparts.

We use DOLPHOT version 2.0 (Dolphin 2000) for the photometric analysis. Following the DOLPHOT manuals for the WFPC2 and WFC3 modules, we photometer the $f t$ (WFC3) and $c 0 m$ (WFPC2) images separately, using the drizzled images produced by the HST pipeline as the reference image. The ULXs are located in crowded regions with high and variable backgrounds, especially NGC 5907 X-1. We find that setting fitsky $=3$, with other parameter values as recommended in the manuals, yields the best results. To select good stars we filter on signal-to-noise ratio $(\mathrm{S} / \mathrm{N}$; must be $>5$ ), sharpness (between -0.3 and +0.3 ), object type (only stars with type 1 , or "good stars"), and photometry flags (only flag values of 0 are selected).

\subsection{ULX Positions}

We determined the positions of the ULXs and other X-ray sources in archival deep Chandra observations of the two galaxies (see Table 2) using the CIAO tool wavdetect. There are two very deep Chandra observations covering M82 X-2, but none of the X-ray sources have unique optical counterparts in the HST images. Instead we register both Chandra observations and the drizzled HST images using Gaia DR2 sources in the field of view. We use the CIAO tool reproject_aspect to register the Chandra observations, only considering X-ray sources with $\mathrm{S} / \mathrm{N}>5$ (194 sources in Obs ID 10542 and 209 sources in Obs ID 10543, excluding M82 X-2 itself). There are 4 and 3 sources with matches in the Gaia catalog for Obs ID 10542 and 10543 , respectively. Given the low number of sources we only calculate the $x$-shift and $y$-shift, leaving the rotation and scale fixed. We adopt the average residual distance between the Gaia and Chandra positions, $0 . .3$, as a measure of the astrometric uncertainty. The positional uncertainty due to localizing the ULX in the Chandra image is negligible $(<0$." 05$)$. The position of M82 X-2 in the two Chandra observations is consistent to within $<0$ " 1 -we adopt the position of the ULX in Obs ID 10543, R.A. = 09:55:51.21, decl. $=+69: 40: 44.1$ (J2000). To register the drizzled $H S T$ images we use the Starlink/GAIA "fit to star positions" tool, with 21 and 19 matched sources for the IR (F160W) and UVIS (F336W) images, respectively. We adopt the rms of the fit as the final astrometric uncertainty: this is $0 . " 12$ for the IR image and $0 . " 04$ for the UVIS image. The uncertainty of the ULX position on the HST images is dominated by the uncertainty in the registration of the Chandra observation and is $0 . ! 3$ for both the IR and UVIS images.

For NGC 5907 we retrieved two Chandra observations from the archive. The shorter one (Obs ID 12987) contains NGC 5907 X-1 but no X-ray sources that can be used for cross-matching with the HST image or other optical catalogs. The longer observation (Obs ID 20830) is a recent DDT observation obtained while NGC 5907 X-1 was in an off-state (Pintore et al. 2018; a previous off-state of this source was observed by Walton et al. 2015). This observation does contain 
Table 2

List of Chandra Observations

\begin{tabular}{lcccc}
\hline \hline Target & Obs ID & Obs Start Date & Inst. & Exp. Time (ks) \\
\hline NGC 5907 & 12987 & 2012 Feb 11 & ACIS-S & 16.0 \\
& 20830 & 2017 Nov 7 & ACIS-S & 51.3 \\
M82 & 10542 & 2009 Jun 24 & ACIS-S & 118.6 \\
& 10543 & 2009 Jul 1 & ACIS-S & 118.5 \\
\hline
\end{tabular}

two X-ray sources with unique counterparts in our WFC3/IR image. Using reproject_aspect, we first register the short Chandra observation to the longer one using 8 common X-ray sources with $\mathrm{S} / \mathrm{N}>4$. The average residual after the shift is 0 ". 23 . We then use the two overlapping sources between the longer Chandra observation and our WFC3/IR image to register the Chandra observations to the HST image. With only two sources we cannot calculate the uncertainty in this step; we conservatively assume an error of 0 !'3. Quadratically adding the two uncertainties gives a total astrometric uncertainty of 0.44 (again, the error in the localization of the ULX in the Chandra image is negligible). The position of the ULXP on the WFC3 image is R.A. $=15: 15: 58.66$, decl. $=+56: 18: 10.3(\mathrm{~J} 2000)$.

The new ULX reported by Pintore et al. (2018, NGC 5907 ULX-2) is visible in Chandra observation 20830. Its position in our WFC3 F160W image is R.A. = 15:16:01.13, decl. $=+56: 17: 51.5(\mathrm{~J} 2000)$, with an astrometric uncertainty of $0 . " 3$ that is mainly due to the registration of Chandra observation 20830 to our HST image.

\subsection{Limiting Magnitudes}

The limiting magnitudes of the HST observations vary across the images due to the local background. We use the DOLPHOT fakestar routine to determine the limiting magnitudes at the positions of the ULXs. In every image we insert 2000 fake stars distributed normally around the position of the ULX, spanning a range of 8 input magnitudes. We then run DOLPHOT in fakestar mode to obtain the photometry of these fake stars. We adopt as the limiting magnitude the faintest magnitude at which $\geqslant 90 \%$ of the fake stars are retrieved with $\mathrm{S} / \mathrm{N} \geqslant 5$. To make sure our photometry is accurate, we also check that the retrieved magnitudes of the fake stars are consistent with their input magnitudes to within $0.3 \mathrm{mag}$. In two cases (the WFC3/ F160W and WFPC2/F814W observations of NGC 5907 X-1) we find that the retrieved magnitudes diverge significantly from the input magnitudes. This is likely due to imperfect modeling of the complex background by DOLPHOT. In these two cases we adopt the faintest magnitude at which $\geqslant 90 \%$ of the fake stars are retrieved within 0.3 mag from their input magnitude, as our limiting magnitude. All magnitude limits are listed in Table 3; we only consider sources brighter than this limit in our analysis.

\section{Results}

\subsection{NGC 5907}

We detect three sources brighter than the limiting magnitude inside the $2 \sigma$ error circle of NGC 5907 X-1 in our F160W image (see the center right panel in Figure 1 and see Table 4). The errors listed are the statistical errors as calculated by DOLPHOT. The brightest source is also detected in the F814W
Table 3

Limiting Magnitudes of HST Images at the Position of the ULXs

\begin{tabular}{lcc}
\hline \hline Source & $H S T$ Filter & Limiting Magnitude \\
\hline M82 X-2 & F225W & 25.1 \\
& F336W & 26.4 \\
& F110W & 18.8 \\
NGC 5907 X-1 & F160W & 18.8 \\
& F450W & 25.5 \\
NGC 5907 ULX-2 & F814W & 23.6 \\
& F160W & 22.0 \\
& F450W & 25.8 \\
& F814W & 25.0 \\
& F160W & 23.7 \\
\hline
\end{tabular}

and F450W images, at $m_{\mathrm{F} 814 \mathrm{~W}} \approx 23.5$ and $m_{\mathrm{F} 450 \mathrm{~W}} \approx 25.0$. No other point sources are detected in the F450W and F814W images.

We detect four sources brighter than the limiting magnitude in the $2 \sigma$ error circle of NGC 5907 ULX-2 in our F160W image (see the right panel in Figure 1 and see Table 4). Only the brightest of these sources is detected in the F450W image. No sources are significantly detected in the F814W image. The Pintore et al. (2018) preferred counterpart is visible in our F160W and F814W images (indicated with a magenta circle in Figure 1), but its position is inconsistent (at $>3 \sigma$ ) with our localization of the ULX. This is due to our smaller localization uncertainty: our $1 \sigma$ uncertainty is 0 ". 3 versus 0 ". 42 for Pintore et al. (2018), as they used a different method to register the Chandra and HST images.

\section{2. $M 82 X-2$}

We detect five sources in the $2 \sigma$ error circle of M82 X-2 in the NIR images, with $17.4 \leqslant m_{\mathrm{F} 110 \mathrm{~W}} \leqslant 18.4$ and $16.0 \leqslant$ $m_{\mathrm{F} 160 \mathrm{~W}} \leqslant 16.8$ (see the right panel in Figure 2 and Table 5). Two of these, as well as seven additional sources that are not detected in the NIR images, are detected in the F336W image (center panel in Figure 2). No point sources are significantly detected in the $\mathrm{F} 225 \mathrm{~W}$ image.

\section{Discussion}

We searched for counterparts to two ULXPs in new and archival HST observations. Both sources are located in crowded regions with high extinction-M82 X-2 in the center of M82, and NGC 5907 X-1 in the dust lane of the edge-on spiral galaxy NGC 5907. We detect multiple potential counterparts to both ULXPs, as well as to a second, recently discovered ULX in NGC 5907. Given the distance modulus to NGC 5907 ( $m-M=31.17 \pm 0.09$, corresponding to a distance of $17.1 \mathrm{Mpc}$; Tully et al. 2013), the absolute magnitudes of the counterparts to NGC $5907 \mathrm{X}-1$ in the F160W filter are $-9.5 \leqslant M_{\mathrm{F} 160 \mathrm{~W}} \leqslant-9.3$. Counterpart 1 is also detected in the F450W and F814W filters with absolute magnitudes of $M_{\mathrm{F} 814 \mathrm{~W}} \approx-7.6$ and $M_{\mathrm{F} 450 \mathrm{~W}} \approx-6.0$, while the other two counterparts are fainter than -7.6 mag in the F814W filter and $-5.7 \mathrm{mag}$ in the F450W filter, respectively. The counterparts to NGC 5907 ULX-2 have $-8.5 \leqslant M_{\mathrm{F} 160 \mathrm{w}} \leqslant$ -7.8 . Counterpart 1 is also detected in the F450W filter with absolute magnitude $M_{\mathrm{F} 450 \mathrm{~W}} \approx-6.5$; the other counterparts are fainter than $-5.4 \mathrm{mag}$ in the F450W filter and all counterparts are fainter than $-6.2 \mathrm{mag}$ in the F814W filter. 


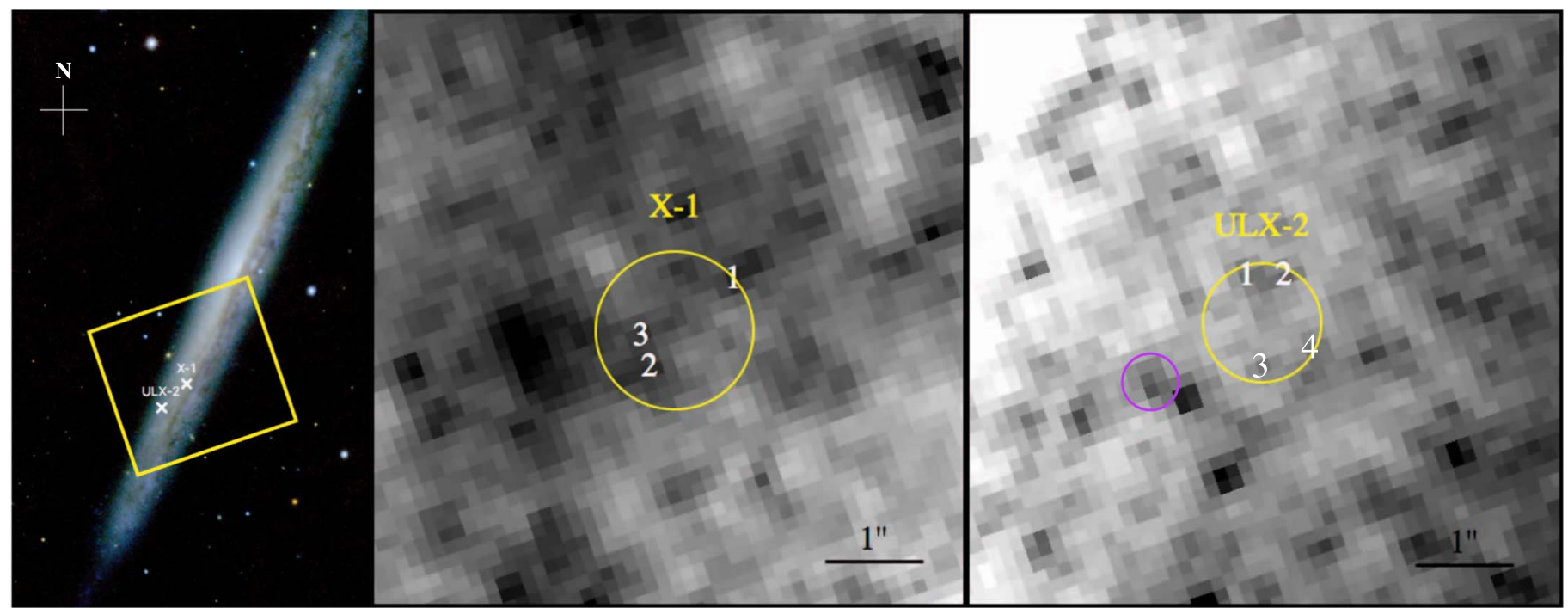

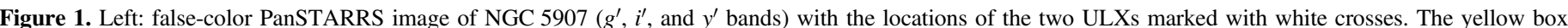

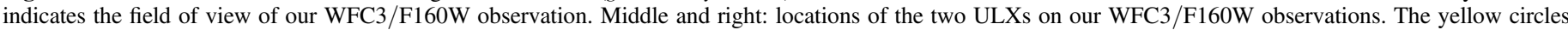

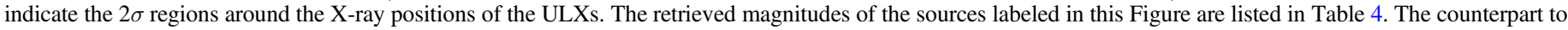
NGC 5907 ULX-2 proposed by Pintore et al. (2018) is indicated with a magenta circle.

Table 4

DOLPHOT Magnitudes of Sources Labeled in Figure 1

\begin{tabular}{lccc}
\hline \hline Source & $m_{\mathrm{F} 160 \mathrm{~W}}$ & $m_{\mathrm{F} 814 \mathrm{~W}}$ & $m_{\mathrm{F} 450 \mathrm{~W}}$ \\
\hline NGC 5907 X-1 & & & \\
1 & $21.61 \pm 0.01$ & $23.53 \pm 0.14$ & $25.0 \pm 0.2$ \\
2 & $21.75 \pm 0.01$ & $>23.6$ & $>25.5$ \\
3 & $21.91 \pm 0.02$ & $>23.6$ & $>25.5$ \\
NGC 5907 ULX-2 & & & \\
1 & $22.68 \pm 0.02$ & $>25.0$ & $24.7 \pm 0.3$ \\
2 & $22.73 \pm 0.02$ & $>25.0$ & $>25.8$ \\
3 & $23.21 \pm 0.03$ & $>25.0$ & $>25.8$ \\
4 & $23.38 \pm 0.04$ & $>25.0$ & $>25.8$ \\
\hline
\end{tabular}

Note. Listed magnitudes are not corrected for extinction.

The five counterparts of M82 X-2 (with a distance modulus of $27.74 \pm 0.08$, corresponding to a distance of $3.5 \mathrm{Mpc}$; Tully et al. 2013) that are detected in the near-IR images have absolute magnitudes $-11.7 \leqslant M_{\mathrm{F} 160 \mathrm{~W}} \leqslant-10.9$ and $-10.4 \leqslant$ $M_{\mathrm{F} 110 \mathrm{~W}} \leqslant-9.2$. Counterparts 1 and 3 are also detected in the F336W image with $M_{\mathrm{F} 336 \mathrm{~W}} \approx-2.8$ and -2.4 , respectively, while the other three are fainter than $-1.3 \mathrm{mag}$ in that band. The other seven counterparts are only detected in the F336W image, with $-3.4 \leqslant M_{\mathrm{F} 336 \mathrm{~W}} \leqslant-1.8$. They are fainter than -8.9 mag in both the F110W and F160W bands. All counterparts are fainter than $-2.6 \mathrm{mag}$ in the $\mathrm{F} 225 \mathrm{~W}$ band.

\subsection{Reddening}

The foreground Galactic extinction in the direction of NGC 5907 is negligible $\left(A_{V} \approx 0.03\right.$, Schlegel et al. 1998). However, NGC 5907 X-1 is located on the dust lane of this edge-on spiral (see Figure 1) and the extinction due to dust and gas in the galaxy itself is significant. The hydrogen column density as measured in the X-ray spectra is $N_{\mathrm{H}}=$ $(0.4-0.9) \times 10^{22} \mathrm{~cm}^{-2}$ (Sutton et al. 2013b; Walton et al. 2015; Fürst et al. 2017). This translates into a $V$-band absorption $A_{V} \approx 1.8-4.1$ (Güver \& Özel 2009). It is not clear from the X-ray observations whether the absorption is variable or not (Fürst et al. 2017). If it is variable, part of this X-ray absorbing material is likely local to the system itself and may not obscure the companion star, and $A_{V}$ is likely closer to the lower end of this range.

NGC 5907 ULX-2 is located farther away from the central dust lane; Pintore et al. (2018) found no local extinction on top of the foreground Galactic absorption from their analysis of the $\mathrm{X}$-ray spectrum of this source.

The foreground Galactic extinction in the direction of M82 is $A_{V}=0.485$ (Schlegel et al. 1998). On top of that there is significant extinction due to gas and dust $\left(A_{V} \geqslant 1.55\right)$ in the core of the galaxy, where M82 X-2 is located (Hutton et al. 2014).

\subsection{Limits on Donor Star Properties from X-Ray Timing}

Assuming circular orbits and accretion via Roche-lobe overflow, the density of the donor star can be determined directly given the orbital period of the binary system and the mass of the accretor, using the equation for the Roche radius (Eggleton 1983) and Kepler's laws. For M82 X-2, the orbital period was reported by Bachetti et al. (2014) as $2.53260 \pm 0.00005$ days, with an eccentricity $\epsilon<0.003$ and a projected semimajor axis $(a \sin (i))$ of $22.225 \pm 0.004$ ls.

For NGC 5907 X-1 these values are not as well constrained. Israel et al. (2017a) performed a likelihood analysis to obtain the orbital parameters of the system (their Figure 2). They found a most-probable period $P_{\text {orb }}=5.3_{-0.9}^{+2.0}$ days with $a \sin (i)=2.5_{-0.8}^{+4.3}$ ls.

For M82 X-2, the lower limit on the donor star mass is $\sim 5$ $M_{\odot}$, assuming a neutron star mass of $1.4 M_{\odot}$ and a system inclination $\leqslant 60^{\circ}$ (Bachetti et al. 2014). For NGC 5907 X-1, the mass function for the most likely parameters reported by Israel et al. (2017a) is $6 \times 10^{-4} M_{\odot}$, implying a lower limit for the donor star of $\sim 0.1 M_{\odot}$. The measurements of Israel et al. (2017a) also allow for a longer orbital period (up to $\sim 21$ days) with a larger projected semimajor axis, which would imply a 


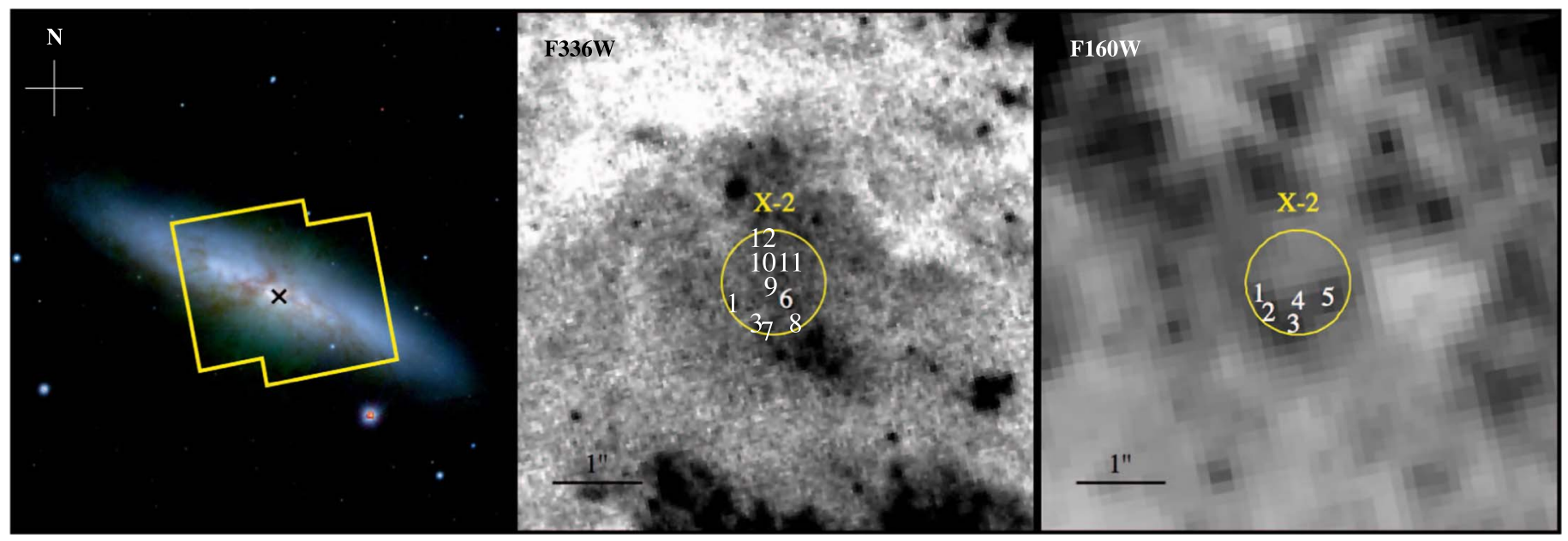

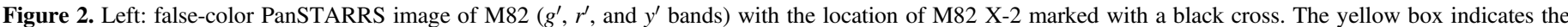

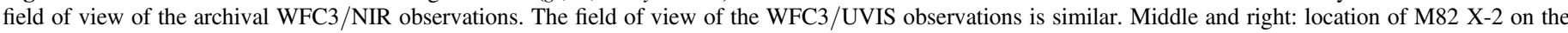

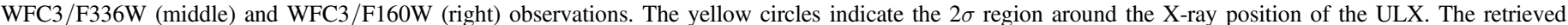
magnitudes of the sources labeled in this Figure are listed in Table 5.

Table 5

DOLPHOT Magnitudes of Sources Labeled in Figure 2

\begin{tabular}{lcccc}
\hline \hline Source & $m_{\mathrm{F} 160 \mathrm{~W}}$ & $m_{110 \mathrm{~W}}$ & $m_{\mathrm{F} 336 \mathrm{~W}}$ & $m_{\mathrm{F} 225 \mathrm{~W}}$ \\
\hline M82 X-2 & & & & \\
1 & $16.719 \pm 0.001$ & $18.411 \pm 0.002$ & $24.9 \pm 0.1$ & $>25.1$ \\
2 & $16.006 \pm 0.001$ & $17.386 \pm 0.001$ & $>26.4$ & $>25.1$ \\
3 & $16.153 \pm 0.001$ & $17.537 \pm 0.001$ & $25.3 \pm 0.2$ & $>25.1$ \\
4 & $16.822 \pm 0.002$ & $18.442 \pm 0.002$ & $>26.4$ & $>25.1$ \\
5 & $16.046 \pm 0.001$ & $17.436 \pm 0.001$ & $>26.4$ & $>25.1$ \\
6 & $>18.8$ & $>18.8$ & $24.39 \pm 0.09$ & $>25.1$ \\
7 & $>18.8$ & $>18.8$ & $25.4 \pm 0.2$ & $>25.1$ \\
8 & $>18.8$ & $>18.8$ & $25.3 \pm 0.2$ & $>25.1$ \\
9 & $>18.8$ & $>18.8$ & $25.6 \pm 0.2$ & $>25.1$ \\
10 & $>18.8$ & $>18.8$ & $25.6 \pm 0.2$ & $>25.1$ \\
11 & $>18.8$ & $>18.8$ & $25.9 \pm 0.3$ & $>25.1$ \\
12 & $>18.8$ & $>18.8$ & $25.7 \pm 0.3$ & $>25.1$ \\
\hline
\end{tabular}

Note. Listed magnitudes are not corrected for extinction.

higher minimum mass for the donor star (e.g., $29 M_{\odot}$ for an orbital period of 18 days and $a \sin (i)=200 \mathrm{ls}$ ).

We plot the relation between allowed masses and radii for M82 X-2 and NGC 5907 X-1 in Figure 3. For comparison, we also show the relation for NGC 7793 P13, a ULXP with a blue supergiant companion with a mass of $18-23 M_{\odot}$ and a radius of 96-125 $R_{\odot}$ (Motch et al. 2014; Fürst et al. 2018). In the background we show evolution tracks of single, non-rotating stars at solar metallicity from the MESA Isochrones and Stellar Tracks (MIST) project (Choi et al. 2016; Dotter 2016). The donor stars in ULXPs do not evolve in isolation, which will impact their evolution tracks (see, e.g., Rappaport et al. 2005; Patruno \& Zampieri 2008, 2010; Ambrosi \& Zampieri 2018). However, comparing the possible masses and radii of the ULXP donors with those of single stars is useful to show the most plausible evolutionary stage of the donor stars in these systems.

From Figure 3 we can see that the donor star of M82 X-2 is most likely just turning off the main sequence (as was also shown by Fragos et al. 2015), while the donor of NGC 5907 $\mathrm{X}-1$ should be a more evolved star in the Hertzsprung gap or on the red giant branch.

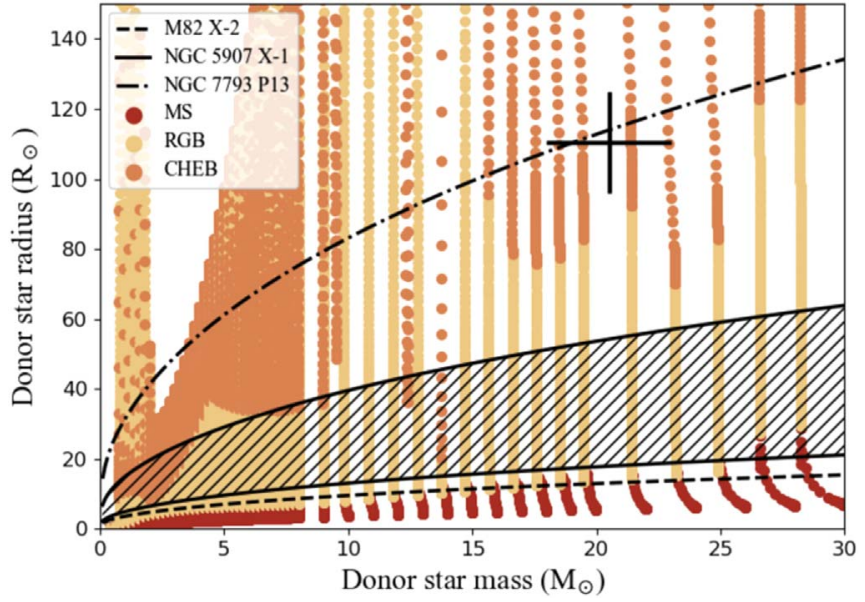

Figure 3. Acceptable values of masses and radii of the donor stars of three ULXPs based on their orbital periods, assuming they accrete through Rochelobe overflow. The lines plotted here assume a neutron star mass of $1.4 M_{\odot}$, but are essentially the same for a neutron star mass of $2 M_{\odot}$. The shaded region indicates the range of allowed orbital periods for NGC 5907 X-1 (4-21 days). The black cross indicates the mass and radius of the donor star of NGC 7793 P13 (Motch et al. 2014; Fürst et al. 2018). In the background are plotted evolution tracks of single stars at solar metallicity obtained from the MIST project. The evolutionary stages plotted here are main sequence (MS), red giant branch stars including the Hertzsprung gap (RGB) and core helium burning (CHEB).

\subsection{Contribution from the Accretion Disk}

The colors and magnitudes of the optical counterparts of ULXs can be significantly affected by irradiation of the donor star and emission from the (supercritical) accretion disk (see, e.g., Copperwheat et al. 2005, 2007; Patruno \& Zampieri 2008, 2010; Ambrosi \& Zampieri 2018 for theoretical work, and Roberts et al. 2011; Grisé et al. 2012 for observations). The theoretical work has so far mostly focused on systems with a (massive) black hole as the accretor, and shows larger effects for more massive black holes; the impact on the optical counterparts of neutron star ULXs may be less pronounced. For example, the optical counterpart of NGC 7793 P13 is affected by irradiation of the donor star, resulting in an observed spectral type that changes with the orbital phase of the system. 
However, its supergiant donor completely dominates the optical emission and there does not appear to be a significant contribution from an accretion disk to the optical light (Motch et al. 2014). Simulations like those of Ambrosi \& Zampieri (2018) for systems with a neutron star accretor would be very useful for assessing the effect of irradiation and the accretion disk on the optical/near-IR emission for ULXPs with nonsupergiant donors. In the following we assume that the optical/ near-IR emission from the ULXPs is dominated by the donor star as is the case for NGC 7793 P13.

\subsection{Limits on Donor Star Properties from Photometry}

To check if the sources we detect in the HST images could be the donor stars of these ULXPs we downloaded synthetic WFC3 and WFPC2 photometry files at solar metallicity with $A_{V}=1.8$ and 4.1 (for NGC $5907 \mathrm{X}-1$ ) and at $[\mathrm{Fe} / \mathrm{H}]=-0.25$ (Nagao et al. 2011) and $A_{V}=2.0$ (for M82 X-2) from the MIST website, as well as WFC3 and WFPC2 photometry at solar metallicity with $A_{V}=0.03$ for NGC 5907 ULX-2. In the case of NGC $5907 \mathrm{X}-1$, all stars with magnitudes consistent with the observed ones are red supergiant stars with radii larger than $400 R_{\odot}$-none of these can be the stellar companion to the ULXP. The limiting magnitude of our WFC3/F160W image is too bright to detect the donor star, due to the high background in this region. The F160W magnitudes and F450W and F814W limits of the sources detected in the error circle of NGC 5907 ULX-2 are consistent with asymptotic giant branch (AGB) stars. Without information on the orbital period and accretor mass in this system, we cannot say anything about the likelihood that one of these sources is the donor star to the ULX.

The near-IR counterparts to M82 X-2 are even brighter, and no single star in the MIST simulations matches these F110W and F160W absolute magnitudes. These sources may be compact star clusters - the resolution of the WFC $3 / \mathrm{IR}$ camera at $1.6 \mu \mathrm{m}$ corresponds to $\sim 3 \mathrm{pc}$ at the distance of M82. The magnitudes and limits of sources that are only detected in the WFC3/UVIS F336W image do match those of single stars with masses and radii consistent with a possible donor of M82 X-2: 10-15 $M_{\odot}$ stars just turning off the main sequence. One of these counterparts could potentially be the donor star of M82 X-2, although it is also possible that the donor is a lower-mass star too faint to be detected in the F336W image. In addition, one of these sources may be the real counterpart, but dominated by irradiation and/or emission from the accretion disk.

\section{Conclusions}

We obtained new WFC3/NIR images of NGC 5907 and used those in conjunction with deep archival WFPC2 images to search for the donor star of NGC 5907 X-1. The sources we detect are too bright to be the donor star of the ULXP, which, based on its orbital period, should be a red giant (see Figure 3 ). The high background at the location of NGC $5907 \mathrm{X}-1$ precludes us from detecting the expected donor star, assuming that the donor dominates the optical/near-IR emission from the system. The recently discovered NGC 5907 ULX-2 also falls within the field of view; we detect four sources in the error circle, with photometry that matches AGB stars. The star suggested to be the counterpart by Pintore et al. (2018) falls outside our $2 \sigma$ error circle.
We also retrieved deep WFC3/NIR and WFC3/UVIS images of M82 X-2 from the HST archive to search for the donor star of this ULXP. The sources detected in the NIR images are too bright to be single stars, but we also detect several sources in the UVIS F336W image whose photometry matches that of 10-15 $M_{\odot}$ stars turning off the main sequence. Such stars have densities consistent with the donor star of M82 X-2, although it is also possible that the donor is a lowermass star fainter than our detection limit or that the optical light is dominated by irradiation and/or emission from the accretion disk.

Characterizing the donor stars of ULXPs is important for testing models of binary evolution. To date, the only system with a spectroscopically characterized donor star is NGC 7793 P13, which hosts a blue supergiant star (Motch et al. 2011, 2014). However, the majority of ULXPs are expected to have lower-mass red giant donor stars (Wiktorowicz et al. 2017). Shorter-period systems such as M82 X-2 and NGC 5907 X-1 may be examples of such lower-mass systems, but the crowded environments and the large distances to these sources make direct detection, especially spectroscopic characterization, unfeasible with currently available instruments. For the nearest ULXs this may change when the first 30-40 m class telescopes become operational.

We would like to thank the anonymous referee whose comments helped to significantly improve the paper.

This work is based on observations made with the NASA/ ESA Hubble Space Telescope, obtained from the Data Archive at the Space Telescope Science Institute, which is operated by the Association of Universities for Research in Astronomy, Inc., under NASA contract NAS 5-26555. These observations are associated with program 15074. Support for Program number 15074 was provided by NASA through a grant from the Space Telescope Science Institute, which is operated by the Association of Universities for Research in Astronomy, Incorporated, under NASA contract NAS 5-26555. The scientific results reported in this article are based in part on data obtained from the Chandra Data Archive. This work has made use of data from the European Space Agency (ESA) mission Gaia (https://www. cosmos.esa.int/gaia), processed by the Gaia Data Processing and Analysis Consortium (DPAC, https://www.cosmos.esa.int/ web/gaia/dpac/consortium). Funding for the DPAC has been provided by national institutions, in particular the institutions participating in the Gaia Multilateral Agreement. The work of DS was carried out at the Jet Propulsion Laboratory, California Institute of Technology, under a contract with NASA.

Facilities: HST (WFPC2 and WFC3), Chandra (ACIS), Gaia.

Software: astropy (Astropy Collaboration et al. 2013), DOLPHOT (Dolphin 2000), MESA (Paxton et al. 2011, 2013, 2015), CIAO (Fruscione et al. 2006).

\section{ORCID iDs}

M. Heida (ib https://orcid.org/0000-0002-1082-7496

M. Brightman (i) https://orcid.org/0000-0002-8147-2602

F. Fürst (i) https://orcid.org/0000-0003-0388-0560

D. Stern (i) https://orcid.org/0000-0003-2686-9241

D. J. Walton (D) https://orcid.org/0000-0001-5819-3552

\section{References}

Ambrosi, E., \& Zampieri, L. 2018, MNRAS, 480, 4918

Astropy Collaboration, Robitaille, T. P., Tollerud, E. J., et al. 2013, A\&A, 558, A33 
Bachetti, M., Harrison, F. A., Walton, D. J., et al. 2014, Natur, 514, 202 Basko, M. M., \& Sunyaev, R. A. 1976, MNRAS, 175, 395

Binder, B., Levesque, E. M., \& Dorn-Wallenstein, T. 2018, ApJ, 863, 141 Brightman, M., Harrison, F. A., Fürst, F., et al. 2018, NatAs, 2, 312

Carpano, S., Haberl, F., Maitra, C., \& Vasilopoulos, G. 2018, MNRAS, 476, L45 Choi, J., Dotter, A., Conroy, C., et al. 2016, ApJ, 823, 102

Colbert, E. J. M., \& Miller, M. C. 2005, in The Tenth Marcel Grossmann Meeting. On Recent Developments in Theoretical and Experimental General Relativity, Gravitation and Relativistic Field Theories, ed. M. Novello, S. Perez Bergliaffa, \& R. Ruffini (Singapore: World Scientific), 530

Copperwheat, C., Cropper, M., Soria, R., \& Wu, K. 2005, MNRAS, 362, 79 Copperwheat, C., Cropper, M., Soria, R., \& Wu, K. 2007, MNRAS, 376, 1407 Dolphin, A. E. 2000, PASP, 112, 1383

Dotter, A. 2016, ApJS, 222, 8

Eggleton, P. P. 1983, ApJ, 268, 368

Esposito, P., Israel, G. L., Milisavljevic, D., et al. 2015, MNRAS, 452, 1112 Fabbiano, G. 1989, ARA\&A, 27, 87

Fragos, T., Linden, T., Kalogera, V., \& Sklias, P. 2015, ApJL, 802, L5

Fruscione, A., McDowell, J. C., Allen, G. E., et al. 2006, Proc. SPIE, 6270, $62701 \mathrm{~V}$

Fürst, F., Walton, D. J., Harrison, F. A., et al. 2016, ApJL, 831, L14

Fürst, F., Walton, D. J., Heida, M., et al. 2018, A\&A, 616, A186

Fürst, F., Walton, D. J., Stern, D., et al. 2017, ApJ, 834, 77

Gladstone, J. C., Copperwheat, C., Heinke, C. O., et al. 2013, ApJS, 206, 14

Gladstone, J. C., Roberts, T. P., \& Done, C. 2009, MNRAS, 397, 1836

Grisé, F., Kaaret, P., Corbel, S., et al. 2012, ApJ, 745, 123

Güver, T., \& Özel, F. 2009, MNRAS, 400, 2050

Heida, M., Jonker, P. G., Torres, M. A. P., et al. 2016, MNRAS, 459, 771

Heida, M., Torres, M. A. P., Jonker, P. G., et al. 2015, MNRAS, 453, 3510

Herold, H. 1979, PhRvD, 19, 2868

Hutton, S., Ferreras, I., Wu, K., et al. 2014, MNRAS, 440, 150

Inoue, Y., Tanaka, Y. T., \& Isobe, N. 2016, MNRAS, 461, 4329

Israel, G. L., Belfiore, A., Stella, L., et al. 2017a, Sci, 355, 817

Israel, G. L., Papitto, A., Esposito, P., et al. 2017b, MNRAS, 466, L48

Jaisawal, G. K., Naik, S., \& Chenevez, J. 2018, MNRAS, 474, 4432

Kaaret, P., Feng, H., \& Roberts, T. P. 2017, ARA\&A, 55, 303

Kissler-Patig, M., Ashman, K. M., Zepf, S. E., \& Freeman, K. C. 1999, AJ, 118,197
Kluzniak, W., \& Lasota, J. P. 2015, MNRAS, 448, L43

Lattimer, J. M. 2012, ARNPS, 62, 485

Lau, R. M., Kasliwal, M. M., Bond, H. E., et al. 2016, ApJ, 830, 142

Lim, S., Hwang, N., \& Lee, M. G. 2013, ApJ, 766, 20

Liu, J.-F., Bregman, J. N., Bai, Y., Justham, S., \& Crowther, P. 2013, Natur, 503, 500

Marchant, P., Langer, N., Podsiadlowski, P., et al. 2017, A\&A, 604, A55

Motch, C., Pakull, M. W., Grisé, F., \& Soria, R. 2011, AN, 332, 367

Motch, C., Pakull, M. W., Soria, R., Grisé, F., \& Pietrzyński, G. 2014, Natur, 514,198

Mushtukov, A. A., Suleimanov, V. F., Tsygankov, S. S., \& Poutanen, J. 2015, MNRAS, 454, 2539

Nagao, T., Maiolino, R., Marconi, A., \& Matsuhara, H. 2011, A\&A, 526, A149

Patruno, A., \& Zampieri, L. 2008, MNRAS, 386, 543

Patruno, A., \& Zampieri, L. 2010, MNRAS, 403, L69

Paxton, B., Bildsten, L., Dotter, A., et al. 2011, ApJS, 192, 3

Paxton, B., Cantiello, M., Arras, P., et al. 2013, ApJS, 208, 4

Paxton, B., Marchant, P., Schwab, J., et al. 2015, ApJS, 220, 15

Pintore, F., Belfiore, A., Novara, G., et al. 2018, MNRAS, 477, L90

Rappaport, S. A., Podsiadlowski, P., \& Pfahl, E. 2005, MNRAS, 356, 401

Roberts, T. P., Gladstone, J. C., Goulding, A. D., et al. 2011, AN, 332, 398

Schlegel, D. J., Finkbeiner, D. P., \& Davis, M. 1998, ApJ, 500, 525

Sutton, A. D., Roberts, T. P., Gladstone, J. C., et al. 2013a, MNRAS, 434, 1702

Sutton, A. D., Roberts, T. P., \& Middleton, M. J. 2013b, MNRAS, 435, 1758

Sutton, A. D., Roberts, T. P., Walton, D. J., Gladstone, J. C., \& Scott, A. E. 2012, MNRAS, 423, 1154

Tsygankov, S. S., Doroshenko, V., Lutovinov, A. A., Mushtukov, A. A., \& Poutanen, J. 2017, A\&A, 605, A39

Tully, R. B., Courtois, H. M., Dolphin, A. E., et al. 2013, AJ, 146, 86

Villar, V. A., Berger, E., Chornock, R., et al. 2016, ApJ, 830, 11

Voss, R., Nielsen, M. T. B., Nelemans, G., Fraser, M., \& Smartt, S. J. 2011, MNRAS, 418, L124

Walton, D. J., Fürst, F., Heida, M., et al. 2018, ApJ, 856, 128

Walton, D. J., Harrison, F. A., Bachetti, M., et al. 2015, ApJ, 799, 122

Wang, S., Liu, J., Bai, Y., \& Guo, J. 2015, ApJL, 812, L34

Wiktorowicz, G., Sobolewska, M., Lasota, J.-P., \& Belczynski, K. 2017, ApJ, 846,17

Wilson-Hodge, C. A., Malacaria, C., Jenke, P. A., et al. 2018, ApJ, 863, 9 